\title{
CROSS SECTIONALLY SIMPLE SPHERES
}

\author{
BY W. T. EATON
}

Communicated by R. H. Bing, April 16, 1968

J. W. Alexander [1] suggested that a 2-sphere $S$ in $E^{\mathbf{z}}$ is tame if each horizontal cross section is either a point or a simple closed curve. It is not clear whether he presumed that his proof was valid for nonpolyhedral spheres, but his proof implies that there is a homeomorphism $h$ of $E^{3}$ onto itself which is invariant on horizontal planes and which takes $S$ onto a round 2-sphere. Bing [6] has described a nonpolyhedral 2-sphere $S$ for which there is no such homeomorphism $h$.

In this paper we give a proof of Alexander's conjecture. The proof, however, is not elementary as it depends indirectly on Dehn's Lemma [8], Bing's Side Approximation Theorem [2], and Bing's Characterization of tame spheres with homeomorphic approximations in their complementary domains [4].

We assume that $S$ lies exactly between the planes $z=1$ and $z=-1$ and we let $J_{t}=S \cap\{(x, y, z) \mid z=t\}$ be the horizontal cross section of $S$ at the $z=t$ plane. Note that $J_{t}$ is a simple closed curve for $-1<t<1$ and $J_{-1}, J_{1}$ are points. We let $D_{t}$ be the disk $J_{t}$ bounds in the $z=t$ plane. The $\epsilon$-neighborhood of a set $X$ is denoted by $N(X, \epsilon)$, Diam $A$ is the diameter of $A$, and $S^{1}$ stands for the standard 1-sphere. If $-1<\alpha<\beta<1$ and $h$ is a homeomorphism of $S^{1} \times[\alpha, \beta]$ into Int $S$ such that

(1) $h(y \times[\alpha, \beta])$ is a vertical line segment for $y \in S^{1}$, and

(2) $h\left(S^{1} \times t\right)$ lies in the plane $z=t$ for $t \in[\alpha, \beta]$,

then $A(h, t)$ denotes the annulus in the $z=t$ plane bounded by $h\left(S^{1} \times t\right)$ and $J_{t}, S(\alpha, \beta)$ denotes the annulus $S \cap\{(x, y, z) \mid \alpha \leqq z \leqq \beta\}$ and $T(h)$ denotes the torus $h\left(S^{1} \times[\alpha, \beta]\right) \cup A(h, \alpha) \cup A(h, \beta) \cup S(\alpha, \beta)$.

Lemma 1. If $t \in(-1,1)$ and $\epsilon>0$ then there are rational numbers $\alpha$ and $\beta$ and $a$ homeomorphism $h: S^{1} \times[\alpha, \beta] \rightarrow \operatorname{Int} S$ such that

(1) $-1<\alpha<t<\beta<1$,

(2) $h(y \times[\alpha, \beta])$ is a vertical line segment for each $y \in S^{1}$,

(3) $h\left(S^{1} \times r\right)$ lies in the horizontal plane $z=r$ for $r \in[\alpha, \beta]$,

(4) $T(h)$ lies in an $\epsilon$-neighborhood of $J_{t}$, and

(5) $h\left(S^{1} \times t\right)$ is homeomorphically within $\epsilon$ of $J_{t}$.

Proof. There is a simple closed curve $J$ in the $z=t$ plane such that $J C$ Int $S, J$ is homeomorphically within $\epsilon$ of $J_{i}$, and the annulus $A$ bounded by $J$ and $J_{t}$ in the $z=t$ plane lies in $N\left(J_{t}, \epsilon\right) . J$ may be moved 
slightly in the vertical direction so there is a $\delta>0$ and a homeomorphism $g: S^{1} \times[t-\delta, t+\delta] \rightarrow$ Int $S$ such that $g(y \times[t-\delta, t+\delta])$ is a vertical line segment for $y \in S^{1}, g\left(S^{1} \times r\right)$ lies in the $z=r$ plane for $r \in[t-\delta, t+\delta]$ and $g\left(S^{1} \times t\right)=J$. Since the annulus $A(g, t)$ lies in $N\left(J_{t}, \epsilon\right)$ and $\lim _{r \rightarrow 1} A(g, r)=A(g, t)$, there are rational numbers $\alpha$ and $\beta$ such that $t-\delta<\alpha<t<\beta<t+\delta$ and $A(g, r) \subset N\left(J_{t}, \epsilon\right)$ if $r \in[\alpha, \beta]$. Take $h=g \mid S^{1} \times[\alpha, \beta]$.

The following lemma is an easy consequence of the Tietze Extension Theorem and the fact that small subsets of $S$ lie in small subdisks on $S$.

Lemma 2. If $D$ is a 2-cell and $\epsilon>0$ then there is a $\delta>0$ such that if $f$ is a map of $\mathrm{Bd} D$ into a $\delta$-subset of Int $S$ then $f$ may be extended to $D$ so that $f(D)$ lies in an $\epsilon$-subset of $\mathrm{Cl}(\operatorname{Int} S)$.

To establish that $S$ is tame from Int $S$ we show that Int $S$ is 1-ULC and use Bing's characterization of tame 2-spheres in $E^{3}$ [3]. That Int $S$ is 1-ULC is an easy consequence of Lemmas 2 and 3.

Lemma 3. If $f$ is a map of 2-cell $D$ into $\mathrm{Cl}(\operatorname{Int} S)$ such that $f(\mathrm{Bd} D)$ $C$ Int $S$ and $\epsilon>0$ then there is a map $g: D \rightarrow \operatorname{Int} S$ such that $f \mid \operatorname{Bd} D$ $=g \mid \operatorname{Bd} D$ and $g(D) \subset N(f(D), \epsilon)$.

Proof. The map $f$ is adjusted in three steps to obtain $g$. In Step I, $f$ is adjusted so that $f(D)$ misses the points $J_{1}$ and $J_{-1}$. In Step II, $f$ is further adjusted so that $f(D) \cap S$ is 0-dimensional and $f(D) \cap J_{r}=\varnothing$ if $r$ is a rational number. The map $f$ is altered in Step III so that $\left.f^{\prime} D\right) \subset$ Int $S$.

Siep I. There is a $\delta>0$ such that Diam $D_{1-\delta}<\epsilon / 3$, Diam $D_{-1+\delta}$ $<\epsilon / 3, D_{1-\delta}$ separates $f(\operatorname{Bd} D)$ from $J_{1}$ on $f(D)$, and $D_{-1+\delta}$ separates $f(\operatorname{Bd} D)$ from $J_{-1}$ on $f(D)$. In two applications of the Tietze Extension Theorem, as indicated in [7, Lemma 1], we may adjust $f$ to obtain a $\operatorname{map} f_{1}: D \rightarrow \mathrm{Cl}(\operatorname{Int} S)$ such that $f\left|\operatorname{Bd} D=f_{1}\right| \operatorname{Bd} D, f_{1}(D) \subset N(f(D), \epsilon / 3)$, $J_{1} \notin f_{1}(D)$, and $J_{-1} \notin f_{1}(D)$.

Step II. Since for $-1<r<1, J_{r}$ is a tame simple closed curve, it follows from the techniques of [5] that there is a map $f_{2}: D \rightarrow \mathrm{Cl}(\operatorname{Int} S)$ such that $f_{2}\left|\mathrm{BD} D=f_{1}\right| \mathrm{Bd}$ this $D, f_{2}(D) \subset N\left(f_{1}(D), \epsilon / 3\right), f_{2}(D) \cap S$ is 0 -dimensional, and $J_{r} \cap f_{2}(D)=\varnothing$ if $r$ is rational.

Step III. For $-1<t<1$ there is a $\delta_{t}>0$ such that if $J$ is homeomor-

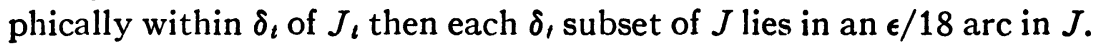
Since $f_{2}(D) \cap S$ is compact and 0-dimensional, for each $-1<t<1$ there is a collection $\mathfrak{C}_{t}$ of disjoint open sets in $E^{3}$ covering $f_{2}(D) \cap S$ such that if $A \in \mathcal{C}_{t}$ then $\operatorname{Diam} A<\delta_{t}$ and $A \cap f(\operatorname{Bd} D)=\varnothing$. Let $\lambda_{t}$ be a positive number which is less than the distance between $f_{2}(D)-\cup \mathfrak{C}_{t}$ 
and $J_{t}$ and which is less than $\epsilon / 36$. By Lemma 1 there are rational numbers $\alpha_{t}$ and $\beta_{t}$ and a homeomorphism $h_{t}: S^{1} \times\left[\alpha_{t}, \beta_{t}\right] \rightarrow \operatorname{Int} S$ such that

(1) $-1<\alpha_{t}<t<\beta_{t}<1$,

(2) $h_{t}\left(y \times\left[\alpha_{t}, \beta_{t}\right]\right)$ is a vertical line segment for each $y \in S^{1}$,

(3) $h_{t}\left(S^{1} \times r\right)$ lies in the horizontal plane $z=r$ for $r \in\left[\alpha_{t}, \beta_{t}\right]$,

(4) $T\left(h_{t}\right)$ lies in an $\lambda_{t}$-neighborhood of $J_{t}$, and

(5) $h_{t}\left(S^{1} \times t\right)$ is homeomorphically within $\delta_{t}$ of $J_{t}$.

A finite number of the tori $\left\{T\left(h_{t}\right)\right\}(-1<t<1)$ suffice to cover $f_{2}(D) \cap S$. This finite collection of tori may be cut apart using horizontal planes $z=r$ with $r$ rational to obtain a new finite collection $\mathfrak{C}$ of disjoint tori which also cover $f_{2}(D) \cap S$. For each $T \in \mathcal{C}$ there exist a number $t$ and rational numbers $u$ and $v$ such that $\alpha_{t}<u<v<\beta_{t}$ and $T=h_{t}\left(S^{1} \times[u, v]\right) \cup A\left(h_{t}, u\right) \cup A\left(h_{t}, v\right) \cup S(u, v)$.

We next show that each component $K$ of $f_{2}(D) \cap(T-S(u, v))$ is contained in the interior of an $\epsilon / 3$-disk in $T-S(u, v)$. By (4) there is an open set in $\mathfrak{e}_{t}$ which contains $K$; consequently, Diam $K<\delta_{t}$. Let $K^{\prime}=\left\{x \in h_{t}\left(S^{1} \times t\right) \mid\right.$ for some $y \in S^{1}, x=h_{t}(y \times t)$ and $\left.K \cap h_{t}(y \times[u, v]) \neq \varnothing\right\}$. It follows that Diam $K^{\prime}<\delta_{t}$ so there is an $\epsilon / 18$-arc $B$ in $h_{t}\left(S^{1} \times t\right)$ that contains $K^{\prime}$. If $M=\left\{x \in S^{1} \mid h_{t}(x \times t) \in B\right\}$ then the disk $E=U_{x \in M} h_{t}(x \times[u, v])$ is of diameter less than $\epsilon / 9$ and contains $K \cap h_{t}\left(S^{1} \times[u, v]\right)$. Since Diam $K \cap I n t A\left(h_{t}, u\right)<\delta_{t}<\epsilon / 9$, $\delta_{t}<$ Diam $h_{t}\left(S^{1} \times u\right)$, and $A\left(h_{t}, u\right)$ lies in a horizontal plane, it follows that $K \cap \operatorname{Int} A\left(h_{t}, u\right)$ does not separate $J_{u}$ from $h_{t}\left(S^{1} \times u\right)$ on $A\left(h_{t}, u\right)$ and consequently lies in an $\epsilon / 9$-subdisk $E_{u}$ of $A\left(h_{t}, u\right)-J_{u} . E_{u}$ may be chosen so that $E_{u} \cap E=\mathrm{Bd} E_{u} \cap \mathrm{Bd} E$ is an arc. Similarly, there is an $\epsilon / 9$-subdisk $E_{v}$ of $A\left(h_{t}, v\right)-J_{v}$ that contains $K \cap A\left(h_{t}, v\right)$ so that $E_{v} \cap E=\mathrm{Bd} E_{v} \cap \mathrm{Bd} E$ is an arc. It follows that $E_{u} \cup E \cup E_{v}$ is an $\epsilon / 3$-subdisk of $T-S(u, v)$ that contains $K$.

Using techniques of the topology of $E^{2}$ it now follows that $(T-S(u, v)) \cap f_{2}(D)$ is covered with a finite collection $D_{T}$ of disjoint $\epsilon / 3$-disks in $T-S(u, v)$. The collection $D=U_{T \in e} D_{T}$ is finite, disjoint, and such that $U_{F \in D} F$ separates $f_{2}(\operatorname{Bd} D)$ from $f_{2}(D) \cap S$ on $f_{2}(D)$. It follows from the Tietze Extension Theorem, as indicated in [7, Lemma 1], that there is a map $g: D \rightarrow \operatorname{Int} S$ such that $g\left|\operatorname{Bd} D=f_{2}\right| \operatorname{Bd} D$ and $g(D) \subset N\left(f_{2}(D), \epsilon / 3\right)$.

From Steps I, II, and III we have $f\left|\operatorname{Bd} D=f_{1}\right| \operatorname{Bd} D=f_{2} \mid \operatorname{Bd} D$ $=g \mid \operatorname{Bd} D$ and $g(D) \subset N(f(D), \epsilon)$.

That $S$ is tame from Int $S$ now follows from Lemma 2, Lemma 3 and Bing's 1-ULC characterization of tame surfaces. Similar techniques are employed to show that $S$ is tame from Ext $S$. Thus we have proved the following theorem suggested by Alexander [1]. 
THEOREM. $A$ 2-sphere $S$ in $E^{3}$ is tame if each horizontal cross section of $S$ is either a simple closed curve or a point.

The author has recently learned that Norman Hosay has also given a proof of this theorem.

\section{REFERENCES}

1. J. W. Alexander, On the subdivision of 3-space by a polyhedron, Proc. Nat. Acad. Sci. U.S.A. 10 (1924), 6-8.

2. R. H. Bing, Approximating surfaces from the side, Ann of Math. (2) 77 (1963), 145-192.

3. - A surface is tame if its complement is 1-ULC, Trans. Amer. Math. Soc. 101 (1961), 294-305.

4. - Conditions under which a surface in $E^{3}$ is tame, Fund. Math. 47 (1959), 105-139.

5. - Pushing a 2-sphere into its complement, Michigan Math. J. 11 (1964), $33-45$.

6. - Spheres in $E^{3}$, Amer. Math. Monthly 71 (1964), 353-364.

7. C. E. Burgess, Characterizations of tame surfaces in $E^{8}$, Trans. Amer. Math. Soc. 114 (1965), 80-97.

8. C. D. Papakyriakojoulos, On Dehn's Lemma and the asphericity of knots, Ann. of Math. (2) 66 (1957), 1-26.

The University of Tennessee, Knoxville, Tennessee 37916 